\title{
Response to Rv2628 latency antigen associates with cured tuberculosis and remote infection
}

\author{
D. Goletti*, O. Butera*, V. Vanini*, F.N. Lauria ${ }^{\#}$, C. Lange ${ }^{\star}$, K.L.M.C. Franken ${ }^{+}$, \\ C. Angeletti ${ }^{\S}$, T.H.M. Ottenhoff ${ }^{+}$and E. Girardi ${ }^{\S}$
}

ABSTRACT: Interferon- $\gamma$ release assays based on region of difference 1 antigens have improved diagnosis of latent tuberculosis infection (LTBI). However, these tests cannot discriminate between recently acquired infection (higher risk of progression to active tuberculosis) and remote LTBI. The objective of the present study was to evaluate the T-cell interferon- $\gamma$ responses to Mycobacterium tuberculosis DosR-regulon-encoded antigens (latency antigens) compared with QuantiFERON TB-Gold In-Tube (QFT-GIT) in subjects at different stages of tuberculosis.

A total of 16 individuals with remote LTBI and 23 with recent infection were studied; 15 controls unexposed to $M$. tuberculosis and 50 patients with active tuberculosis and 45 with cured tuberculosis were also analysed.

The results indicated that subjects with remote LTBI showed significantly higher whole-blood interferon- $\gamma$ responses to $M$. tuberculosis latency antigen Rv2628 than did individuals with recent infection, active tuberculosis and controls $(p<0.003)$, whereas no significant differences between these groups were found for other latency antigens tested (Rv2626c, Rv2627c, Rv2031c and Rv2032). The proportion of responders to Rv2628 was five-fold higher among QFT-GIT-positiveindividuals with remote infection than among those with recently acquired infection.

These data suggest that responses to $M$. tuberculosis latency antigen Rv2628 may associate with immune-mediated protection against tuberculosis. In contact-tracing investigations, these preliminary data may differentiate recent (positive QFT-GIT results without responses to Rv2628) from remote infection (positive to both tests).

KEYWORDS: Diagnostics, dormancy, recent tuberculosis infection, remote latent tuberculosis infection, tuberculosis

ccording to surveys with tuberculin skin tests (TSTs), the World Health Organization estimated that approximately a third of the world's population is latently infected with Mycobacterium tuberculosis. This enormous reservoir of latent tuberculosis (TB) infection (LTBI), from which most cases of active TB arise, embodies a major obstacle to achieving worldwide control over TB [1].

The introduction of T-cell-based interferon (IFN)- $\gamma$ release assays (IGRAs), using antigens belonging to $M$. tuberculosis region of difference 1 (including 6-kDa early secreted antigenic target (ESAT-6) and culture filtrate protein (CFP) 10), may represent a significant step towards improved LTBI diagnosis [2-4]. There is evidence that commercial IGRAs are highly sensitive for detecting LTBI [2], and have a high specificity that is unaffected by bacille Calmette-Guérin (BCG) vaccination. Although
IGRAs were designed as assays for LTBI, these tests do not discriminate between active disease and LTBI [5, 6]. Moreover, IGRAs do not distinguish between a recently acquired infection and remote LTBI [7]. This information may have important clinical implications, since recently acquired infection carries a significantly increased risk of progression to active disease compared to remote LTBI [8].

During LTBI, tubercle bacilli are contained within immune-mediated granulomas [9]. It is thought that persisting tubercle bacilli are subjected to nutrient and oxygen deprivation [10,11]. As part of the adaptive response of $M$. tuberculosis to hypoxia, expression of the DosR regulon is observed. The functions of most DosR-regulonencoded proteins, hereafter referred to as latency antigens, are unknown [12, 13]. However, it has recently been shown that certain latency antigens
AFFILIATIONS

${ }^{*}$ Translational Research Unit, Dept of Epidemiology and Preclinical Research,

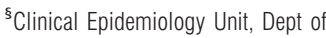
Epidemiology and Preclinical Research,

${ }^{*}$ Clinical Dept, National Institute for Infectious Diseases L. Spallanzani,

Istituto Di Ricovero e Cura a Carattere Scientifico, Rome, Italy.

"Clinical Infectious Diseases, Borstel Research Center, Borstel, Germany. +Dept of Infectious Diseases, Leiden University Medical Center, Leiden, the Netherlands.

CORRESPONDENCE

D. Goletti

Istituto Nazionale per le Malattie Infettive "L. Spallanzani' Via Portuense 292 Rome 00149 Italy E-mail: delia.goletti@inmi.it

Received

Sept 042009

Accepted after revision:

Nov 112009

First published online:

Nov 192009 
are recognised more frequently in TST-positive individuals without active TB than in diseased patients, whereas the opposite profile was found for CFP-10 and ESAT-6 [13-17]. In particular, it has been shown that Rv2627c, Rv2628 and Rv3407 induce strong long-term IFN- $\gamma$ responses in TST-positive individuals. These studies, in which an experimental setting involving long-term (6-7 days) stimulation of frozen/fresh peripheral blood mononuclear cells (PBMCs) or whole blood was used, suggest a contribution of immune responses against the latency antigens to controlling LTBI.

Lately, both short- and long-term whole-blood IGRAs [17-19] have been shown to be accurate in detecting immune responses to $M$. tuberculosis [2], and thus useful for diagnostic purposes. Therefore, the objective of this proof-of-principle study was to evaluate the whole-blood IFN- $\gamma$ response to the recently described antigens of latency (Rv2626c, Rv2627c, Rv2628, Rv2031c and Rv2032) in day-1 (short-term response) and day-7 (long-term response) assays in the different stages of TB. Long-term response was assessed to be in line with results reported in the literature. Subjects with remote and recent infection, individuals with cured TB, patients with active TB and controls not exposed to $M$. tuberculosis were studied. All enrolled individuals were also tested using a commercial IGRA (QuantiFERON-TB Gold In-Tube (QFT-GIT)), with purified protein derivative (PPD) as control antigen.

\section{MATERIAL AND METHODS}

\section{Study population}

The following individuals were enrolled. 1) Controls: individuals with no risk of $M$. tuberculosis infection who tested TST-negative and QFT-GIT-negative. 2) Recent infection: individuals who reported household or equivalent close contact (work) [20] with smear-positive pulmonary TB patients in the previous 3 months, tested TST- and QFT-GITpositive, with negative chest radiographic results for pulmonary lesions and no prior preventive therapy. 3) Remote LTBI: individuals who tested TST- and QFT-GIT-positive and reported household or equivalent contact with patients with smear-positive pulmonary $\mathrm{TB} \geqslant 3$ yrs before enrolment and who did not receive preventive therapy. 4) Cured TB: individuals with culture-positive pulmonary TB who had completed a 6-month course of treatment and were culturenegative upon treatment completion. The patients were evaluated at completion of therapy. 5) Active TB: individuals diagnosed with TB (either with a positive culture for $M$. tuberculosis from sputa or with positive M. tuberculosis-specific RNA amplification (MTD Test; Gen-Probe, San Diego, CA, USA) results from biopsy specimens and/or biological fluids) who started specific treatment $<8$ days before enrolment. Remote and recent infection subjects were comparable in terms of exposure (they all were close contacts). Individuals who tested positive to HIV antibody (three subjects) or were on immunosuppressive drugs (two subjects) were not included in the study. Upon enrolment, demographic and epidemiological information was collected through a structured questionnaire. The study was approved by the ethics committee of the National Institute for Infectious Diseases L. Spallanzani (Rome, Italy) and all enrolled individuals provided written informed consent.

\section{M. tuberculosis antigens}

DosR-regulon-encoded genes were selected on the basis of their RNA expression level in microarray experiments [13]. Genes were cloned and the proteins overexpressed in Escherichia coli and purified as previously described [21].

\section{Tuberculin skin test}

TSTs were administered by the Mantoux procedure using 5 IU of PPD (Chiron, Siena, Italy). Results were read after $72 \mathrm{~h}$. Indurations of $\geqslant 5$ or $\geqslant 10 \mathrm{~mm}$ were scored positive for recent infection or for the other conditions, respectively [22].

\section{Whole-blood ELISAs with M. tuberculosis antigens expressed during latency}

1-day (short-term) response

Heparinised whole blood was seeded in a 48-well plate (Corning Costar; Corning, Inc., New York, NY, USA) at $0.5 \mathrm{~mL} \cdot$ well $^{-1}$ and treated with phytohaemagglutinin at $5 \mu \mathrm{g} \cdot \mathrm{mL}^{-1}$ (Sigma, St Louis, MO, USA), Rv2628 and Rv2031c at $1 \mu \mathrm{g} \cdot \mathrm{mL}^{-1}$, and PPD (batch RT 47 , Statens Serum Institute, Copenhagen, Denmark), Rv2626c, Rv2627c and Rv2032 at $5 \mu \mathrm{g} \cdot \mathrm{mL}^{-1}$. These concentrations were chosen after titration of the various reagents (data not shown). Samples were then incubated for $24 \mathrm{~h}$. At day 1, plasma was harvested and stored at $-20^{\circ} \mathrm{C}$ until tested.

\section{7-day (long-term) response}

Previously described methodology was used [17, 18]. Briefly, at the day of collection, an aliquot of heparinised blood was diluted five-fold using RPMI 1640 supplemented with penicillin, streptomycin and $2 \mathrm{mM}$ L-glutamine (all from Euroclone, Paignton, UK), plated into 48-well plates (Corning Costar) and stimulated as above described. The day-7 diluted plasma was harvested following incubation at $37^{\circ} \mathrm{C}$ and stored at $-20^{\circ} \mathrm{C}$ until use.

\section{IFN- $\gamma$ determination}

IFN- $\gamma$ from day-1 and day-7 plasma was evaluated using a commercial ELISA (CMI; Cellestis, Carnegie, Australia) and data are presented in international units per millilitre, after subtraction of the appropriate control. For concentrations above that of the highest standard, the assay was repeated with diluted plasma samples.

\section{Commercially available IGRA}

QFT-GIT (Cellestis) was performed and its results were scored as indicated by the manufacturer (the cut-off value for a positive test was $0.35 \mathrm{IU} \cdot \mathrm{mL}^{-1}$ ).

\section{Statistical analysis}

The main outcome of the study was the evaluation of IFN- $\gamma$ production in response to antigenic stimulation, expressed using continuous (international units per millilitre) or dichotomous (positive/negative) measures. For continuous measures, mean \pm SD IFN- $\gamma$ production was calculated, an unpaired t-test was used for pairwise comparisons and ANOVA was used to compare means among the various groups. The results were confirmed by evaluation with the Mann-Whitney test for pairwise comparisons and the Kruskal-Wallis test for multiple comparisons after calculation of the median and interquartile range (data not shown). For pairwise comparisons, a post hoc 
TABLE 1 Demographic and clinical characteristics of subjects enrolled in the study

\begin{tabular}{|c|c|c|c|c|c|c|}
\hline Subjects $\mathbf{n}$ & 149 & 15 & 16 & 23 & 50 & 45 \\
\hline Female sex & 69 (46.3) & $10(66.7)$ & $4(25.0)$ & $12(52.2)$ & $21(42.2)$ & $22(48.9)$ \\
\hline$B C G$ vaccinated & $95(63.8)$ & $6(40.0)$ & $3(18.3)$ & $14(60.9)$ & $41(82.0)$ & $31(68.9)$ \\
\hline \multicolumn{7}{|l|}{ Origin } \\
\hline Asia & $18(12.1)$ & & $1(6.3)$ & $3(13.0)$ & $10(20.0)$ & $4(8.9)$ \\
\hline Africa & $10(6.7)$ & & & $2(8.7)$ & $4(8.0)$ & $4(8.9)$ \\
\hline Latin America & $6(4.0)$ & $1(6.7)$ & & & $3(6.0)$ & $2(4.4)$ \\
\hline \multicolumn{7}{|l|}{ Confirmed TB localisation } \\
\hline Pulmonary & $41(27.5)$ & & & & $41(82.0)$ & \\
\hline
\end{tabular}

Data are presented as mean \pm SD or $n(\%)$, unless otherwise stated. LTBI: latent tuberculosis (TB) infection; BCG: bacille Calmette-Guérin; TST: tuberculin skin test; ND: not done.

analysis with Bonferroni correction was used, and differences were considered significant at $\mathrm{p}$-values of $\leqslant 0.05$.

For dichotomous measures, the Chi-squared test was used. For pairwise comparisons, the Bonferroni correction was used, and differences were considered significant at $p$-values of $\leqslant 0.05$. The cut-off value for definition of positivity of the whole-blood assay based on Rv2628 was defined by receiver operating characteristic analysis. SPSS version 14 for Windows (SPSS Italia, Bologna, Italy) and Prism 4 software (Graphpad Software, San Diego, CA, USA) were used in the analysis.

\section{RESULTS}

\section{Population characteristics}

A total of 15 controls and 16 individuals with remote LTBI, 50 with active TB, 45 with cured TB and 23 with recent infection were studied. Age, sex, BCG vaccination, origin and TST results are reported among the different groups (table 1). The majority were male and BCG-vaccinated, almost $40 \%$ were from Western Europe and pulmonary localisation was that most represented in those with active TB.

\section{In vitro short-term IFN- $\gamma$ response to $\mathrm{M}$. tuberculosis latency antigens: quantitative analysis}

The IFN- $\gamma$-specific responses to Rv2628, Rv2626c, Rv2627c, Rv2031c and Rv2032 were evaluated in the various groups. All of the subjects responded to the mitogen: controls (mean $\pm \mathrm{SD}$ $\left.15.7 \pm 7.6 \mathrm{IU} \cdot \mathrm{mL}^{-1}\right)$, recent infection $\left(15.1 \pm 8.4 \mathrm{IU} \cdot \mathrm{mL}^{-1}\right)$, remote LTBI $\left(15.7 \pm 8.4 \mathrm{IU} \cdot \mathrm{mL}^{-1}\right)$, active TB $\left(10.0 \pm 8.6 \mathrm{IU} \cdot \mathrm{mL}^{-1}\right)$, and cured TB $\left(15.3 \pm 10.9 \mathrm{IU} \cdot \mathrm{mL}^{-1}\right)$. A significant difference was found on overall comparison among the various groups $(p=0.026)$, whereas no significant difference was found in pairwise comparisons.

Analysing the IFN- $\gamma$ response to Rv2628, a significant difference was found in the overall comparison among the different groups $(p<0.001)$. For pairwise comparisons, the response to Rv2628 (fig. 1) was significantly higher in those with remote LTBI $\left(5.3 \pm 5.9 \mathrm{IU} \cdot \mathrm{mL}^{-1}\right)$ than in recent infection $\left(0.3 \pm 0.5 \mathrm{IU} \cdot \mathrm{mL}^{-1}\right.$, $\mathrm{p}=0.001)$ and active TB $\left(1.1 \pm 3.4 \mathrm{IU} \cdot \mathrm{mL}^{-1} ; \mathrm{p}=0.002\right)$, whereas no significant difference was observed in comparison with cured TB $\left(3.1 \pm 5.1 \mathrm{IU} \cdot \mathrm{mL}^{-1} ; \mathrm{p}=0.547\right)$. Moreover, the difference between cured TB and recent infection $(p=0.056)$ was close to significance. Finally, a significant difference was found between controls $\left(0.1 \pm 0.1 \mathrm{IU} \cdot \mathrm{mL}^{-1}\right)$ and subjects with remote LTBI $(\mathrm{p}=0.003)$.

Regarding the other latency antigens tested, Rv2626c, Rv2627c, Rv2031c and Rv2032, no significant IFN- $\gamma$ differences were

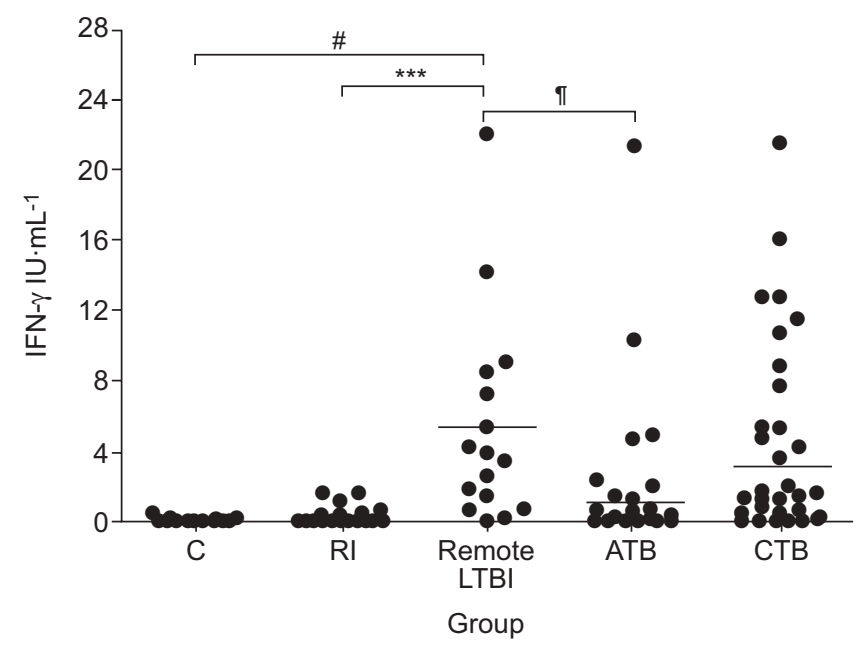

FIGURE 1. Interferon (IFN)- $\gamma$ response to Mycobacterium tuberculosis Rv2628 by short-term (1-day) stimulation of whole blood in controls (C), remote latent tuberculosis (TB) infection (LTBI), recent infection (RI), patients with TB disease (active TB (ATB)) and successfully treated TB patients (cured TB (CTB)). Remote LTBI and RI subjects were selected to be positive on QuantiFERON TB-Gold InTube. Horizontal bars represent means. Responses were compared using ANOVA with Bonferroni correction for pairwise comparisons. ${ }^{* *}: p<0.001 ;{ }^{*}: p<0.003$; ?: $p<0.002$. 

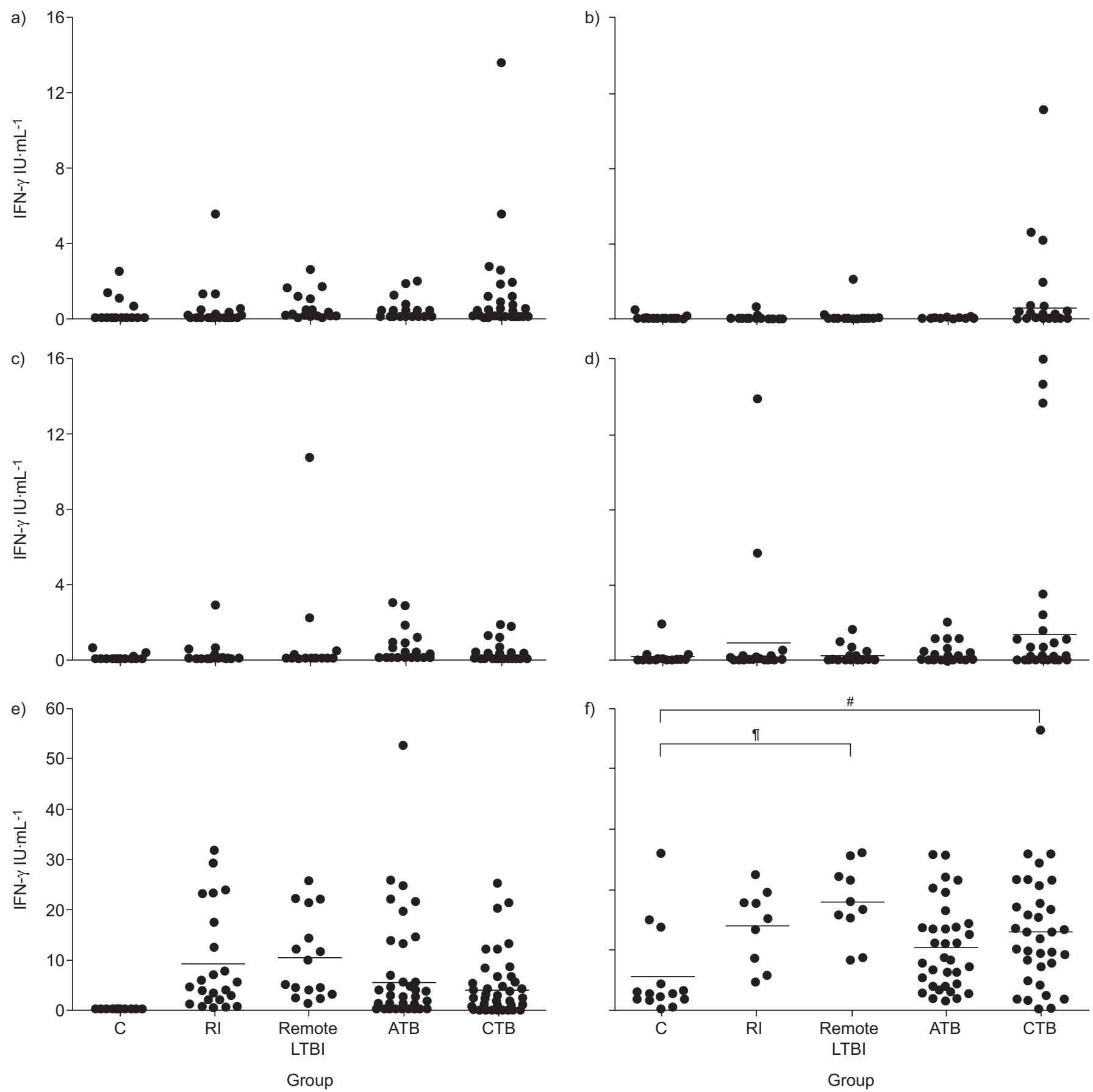

FIGURE 2. Interferon (IFN)- $\gamma$ response to Mycobacterium tuberculosis: a) Rv2626c; b) Rv2627c; c) Rv2031c; d) Rv2032; e) QuantiFERON TB-Gold In-Tube (QFT-GIT); and f) purified protein derivative by short-term (1-day) stimulation of whole blood in controls (C), remote latent tuberculosis (TB) infection (LTBI), recent infection (RI), patients with TB disease (active TB (ATB)) and successfully treated TB patients (cured TB (CTB)). Remote LTBI and RI subjects were selected to be positive on QFT-GIT. Horizontal bars represent means. Responses were compared using ANOVA with Bonferroni correction for pairwise comparisons. ${ }^{\#:} p<0.04 ;{ }^{\natural}: p<0.004$.

found, considering either all of the groups simultaneously or performing pairwise comparisons (fig. 2a-d).

As an internal control, IFN- $\gamma$ response was evaluated in the QFT-GIT, whose antigens are secreted antigens and associated with the replicative status of the mycobacteria, and to PPD, a nonspecific mixture of mycobacterial antigens. Quantitative responses measured by QFT-GIT are shown in figure 2e. Among individuals with LTBI, who were selected on the basis of a QFT-GIT-positive response, no significant difference was observed by comparing recent infection $\left(9.2 \pm 10.0 \mathrm{IU} \cdot \mathrm{mL}^{-1}\right)$ to remote LTBI $\left(10.3 \pm 8.4 \mathrm{IU} \cdot \mathrm{mL}^{-1}\right)$. The proportion of individuals with a positive response to QFT-GIT was similar between active TB (36 out of $50 ; 72.0 \%$ ) and cured TB (31 out of $45 ; 68.9 \%$ ), and 
no significant difference was observed when the responses in these two groups were compared in quantitative terms $(5.5 \pm 9.8$ and $4.0 \pm 6.0 \mathrm{IU} \cdot \mathrm{mL}^{-1}$, respectively).

Response to PPD was analysed in a large proportion of the subjects enrolled (14 out of 15 controls, eight out of 23 with recent infection, 10 out of 16 with remote LTBI, 34 out of 50 with active TB, and 34 out of 45 with cured TB). When quantitative data were analysed, a significant difference in the response to PPD was found when considering all of the groups simultaneously $(p=0.004)$. For pairwise comparisons, a significant difference was found between controls $\left(5.5 \pm 7.4 \mathrm{IU} \cdot \mathrm{mL}^{-1}\right)$ and remote LTBI $\left(17.7 \pm 6.2 \mathrm{IU} \cdot \mathrm{mL}^{-1} ; \mathrm{p}=0.004\right)$ and between controls and cured TB $\left(13.0 \pm 9.6 \mathrm{IU} \cdot \mathrm{mL}^{-1} ; \mathrm{p}=0.041\right)$ (fig. $\left.2 \mathrm{f}\right)$. No significant differences were found between controls and recent infection $(13.8 \pm 6.3)$ or between controls and active TB $\left(10.3 \pm 7.1 \mathrm{IU} \cdot \mathrm{mL}^{-1}\right)$, active and cured TB, or remote LTBI and recent infection.

Taken together, these data suggest that IFN- $\gamma$ responses to Rv2628 are most frequently found in those able to control $M$. tuberculosis replication, either naturally (remote LTBI) or following chemotherapy (cured TB), compared to those with active $M$. tuberculosis replication, either controlled, but acute (recent infection), or uncontrolled (active disease). These responses are different from those found for all other antigens evaluated, including the remaining $M$. tuberculosis latency antigens (Rv2626c, Rv2627c, Rv2031c and Rv2032) and QFTGIT antigens (ESAT-6, CFP-10 and Rv2654 (TB7.7)), as well as PPD, since these responses did not differ significantly among the above-mentioned groups.

\section{In vitro short-term IFN- $\gamma$ response to $\mathrm{M}$. tuberculosis latency antigen Rv2628: qualitative analysis}

Based on the significant difference found in the quantitative analysis, a receiver operating characteristic analysis was performed for the response to Rv2628 in order to evaluate its potential use in discriminating the different stages of TB. In this analysis, remote LTBI and active TB were used as comparator groups because they represented the best examples of $M$. tuberculosis containment and active replication, respectively. Significant results were obtained for area under the curve analysis $(0.85 ; 95 \%$ CI $0.75-0.96 ; p<0.001)$. For scoring purposes a cut-off maximising the sum of sensitivity and specificity was chosen. It was found that a cut-off of 0.5 predicted LTBI with 76.0\% sensitivity (95\% CI 61.8-86.9\%) and $87.5 \%$ specificity (95\% CI 61.6-98.4\%).

Finally, based on the cut-off value found for the responses to Rv2628, the results were scored as negative and positive. A significant difference was found among the various groups $(p<0.001)$. As shown in table 2, the highest proportion of positive results was found among remote LTBI (14 out of 16; $87.5 \%$ ), which was positive to QFT-GIT by definition, followed by cured TB (24 out of $45 ; 53.3 \%$ ), which was $68.9 \%$ positive to QFT-GIT (table 3). Lower proportions of positive response to Rv2628 were found in individuals with active TB (12 out of 50; $24.0 \%$ ) and in those with recent infection (four out of 23; $17.3 \%)$; the proportion of positive responses to QFT-GIT in these two groups were 72.0 and $100 \%$ (by definition), respectively. None of the controls responded to Rv2628, and were negative to QFT-GIT by definition.

A pairwise comparison of positivity rate for Rv2628 in the five groups of individuals enrolled is also shown in table 2. Proportions of positive responses among remote LTBI were significantly higher than those recorded among active TB, recent infection and controls, whereas the difference from cured LTBI was nonsignificant. A significant difference was also found between cured TB compared to recent infection or controls, whereas the difference between cured and active TB $(p=0.056)$ was close to significance.

\section{In vitro long-term IFN- $\gamma$ response to M. tuberculosis latency antigens}

The long-term IFN- $\gamma$ response was assessed to be in line with results reported in the literature, although performed in different experimental settings [17-20]. Long-term IFN- $\gamma$ response to Rv2628 was significantly different among the groups analysed simultaneously $(\mathrm{p}<0.001)$. Moreover, this response was significantly higher in those with remote LTBI $\left(12.1 \pm 13.0 \mathrm{IU} \cdot \mathrm{mL}^{-1}\right)$ than among those with recent infection $\left(2.9 \pm 5.3 \mathrm{IU} \cdot \mathrm{mL}^{-1} ; \quad \mathrm{p}=0.002\right)$, controls $\left(0.55 \pm 1.6 \mathrm{IU} \cdot \mathrm{mL}^{-1}\right.$ $\mathrm{p}<0.001)$ and active TB $\left(1.7 \pm 3.2 \mathrm{IU} \cdot \mathrm{mL}^{-1} ; \mathrm{p}<0.001\right)$. Similar to the findings reported for the short-term responses, a significant difference was found between the response found in active TB compared to cured TB $(p=0.019)$, and the difference found between cured TB and controls was close to significance levels $(\mathrm{p}<0.058)$. Results similar to those described for the short-term responses were found when analysing the qualitative data (data not shown). Regarding the other latency antigens (Rv2626c,

\begin{tabular}{|c|c|c|c|c|c|}
\hline & Rv2628 positivity n/N (\%) & Cured TB & Active TB & Recent infection & Controls \\
\hline Remote LTBI & $14 / 16(87.5)$ & 0.178 & $<0.001$ & $<0.001$ & $<0.001$ \\
\hline Cured TB & 24/45 (53.3) & & 0.056 & 0.048 & 0.001 \\
\hline Active TB & $12 / 50(24.0)$ & & & 1.000 & 0.537 \\
\hline
\end{tabular}




\begin{tabular}{|c|c|c|c|}
\hline \multirow[t]{2}{*}{ TABLE 3} & \multicolumn{3}{|c|}{$\begin{array}{l}\text { Accuracy of the response to QuantiFERON TB- } \\
\text { Gold In-Tube (QFT-GIT) and Rv2628 in the } \\
\text { diagnosis of tuberculosis (TB) status }\end{array}$} \\
\hline & QFT-GIT & Rv2628 & p-value ${ }^{\#}$ \\
\hline Active TB & $36 / 50(72.0)$ & $12 / 50(24.0)$ & $<0.0001$ \\
\hline Cured TВ & $31 / 45(68.9)$ & 24/45 (53.3) & 0.1 \\
\hline \multicolumn{4}{|c|}{$\begin{array}{l}\text { Data are presented as } n / \mathrm{N}(\%) \text {, where } \mathrm{N} \text { is the number of subjects in the group, } \\
\text { unless otherwise indicated. }{ }^{*} \text { : the hypothesis of equal proportion was tested } \\
\text { using Fisher's exact test. }\end{array}$} \\
\hline
\end{tabular}

Rv2627c, Rv2031c and Rv2032), no significant difference in terms of IFN- $\gamma$ release was found for all of the comparisons performed (data not shown). Regarding PPD, a significant difference was found in the overall comparison among the different groups $(p=0.001)$. For pairwise comparisons, significant differences were found only between controls $\left(8.6 \pm 7.4 \mathrm{IU} \cdot \mathrm{mL}^{-1}\right)$ and remote LTBI $\left(21.1 \pm 4.6 \mathrm{IU} \cdot \mathrm{mL}^{-1} ; \mathrm{p}=0.001\right)$, and controls and cured TB $\left(6.8 \pm 9.0 \mathrm{IU} \cdot \mathrm{mL}^{-1} ; \mathrm{p}=0.007\right)$.

\section{DISCUSSION}

In the present proof-of-principle study, the T-cell IFN- $\gamma$ responses of individuals at different stages of $M$. tuberculosis infection/disease and uninfected controls to a series of proteins expressed during M. tuberculosis dormancy (Rv2626c, Rv2627c, Rv2628, Rv2031c, Rv2032) were analysed. The response to $M$. tuberculosis early-phase secreted antigens (ESAT-6, CFP-10 and TB7.7), as assessed by QFT-GIT, and to a mixture of mycobacterial antigens, as present in PPD, were also evaluated.

Among all of the antigens evaluated, Rv2628 induced a significantly higher IFN- $\gamma$ response, and increased proportions of positive responses to this antigen were found in remote LTBI and cured TB compared to recent infection and active TB. Among QFT-GIT-positive individuals with remote LTBI, the proportion of responders to Rv2628 was almost five-fold higher compared to QFT-GIT-positive individuals recently infected with M. tuberculosis (87.5 versus $17.3 \%$ respectively). When analysed in quantitative terms, similar results were obtained using long- or short-term assay. However, the wholeblood, day-1 format appears more appealing for potential use as a diagnostic tool. Taken together, these results suggest that the response to Rv2628 can be used to characterise LTBI as a remote versus recently acquired infection. This can be helpful in the clinical management of recently acquired infection, since persons with this condition may receive the greatest benefit from preventive therapy [8].

The M. tuberculosis latency antigens used in the present study are encoded by the $M$. tuberculosis DosR regulon, which has been shown to play a critical role in preparing $M$. tuberculosis for the metabolic downshift associated with bacterial dormancy [23, 24]. At present, the exact role of DosR regulon products in human $M$. tuberculosis infection is incompletely understood. Rv2031c, the archetypal DosR regulon product, is a small heat shock protein, and cellular immune responses to Rv2031c have been observed in individuals with LTBI [25]. More recently, LEYTEN et al. [14] studied human T-cell responses to a set of the 25 DosR encoded proteins, using long-term cultured thawed PBMCs. The results provided the first support for the immunogenicity of DosR regulon proteins in human PBMC stimulation assays of TST-positive individuals. Subsequently, the latency antigens Rv1733c, Rv2029c, Rv2627c and Rv2628 were identified as the most frequently recognised antigens in latently infected individuals [14, 16]. Similarly, the IFN- $\gamma$ response to Rv3407 has recently been shown to be a response specific to LTBI [15]. The present results confirm that a cellular immune response to these latency antigens can be found in vitro, and show that this response can also be efficiently detected by means of a wholeblood 1-day assay. It was also found that the response to Rv2628 antigen was more clearly associated with a latent infection, as previously shown using other experimental approaches by LEYTEN et al. [14] and BLACK et al. [16] and confirmed by RoupIE et al. [26] in mice. However, from all of the studies reported, it is presently unclear why the response to Rv2628 may provide better discrimination between the different stages of TB infection. Among the studies performed on human samples [14-17], SCHUCK et al. [15] did not show any immunogenic activity of Rv2628. This may probably be due to the selection of LTBI subjects that have not been clearly defined as recently or remotely infected.

Detectable responses to $M$. tuberculosis latency antigens, although limited, were also found in active TB patients. This is probably due to the fact that these antigens are expressed in response to immune and physiological stress experienced by the bacteria, which eventually result in latency, and immune responses to the antigens induced during this phase may remain detectable [23, 26, 27].

As previously shown [14], low levels of recognition of the latency antigens $\operatorname{Rv} 2626 c$ and $\operatorname{Rv} 2627 c$ by some of the uninfected controls were found and were uncorrelated with BCG vaccination (data not shown). This result may most probably be explained by exposure to mycobacteria other than M. tuberculosis that have a similar dormancy regulon [28-30]. DosR-regulon-encoded antigens are also present in BCG, but, in the present study, vaccination does not seem to be associated with induction of responses to Rv2628 in either controls or subjects with recent infection, as previously reported [31].

The present study has a number of limitations. The individuals with recent infection were the contacts of sputum-smearpositive TB patients within the last 3 months. By definition, they were all TST-positive. However, TST conversion was documented in only eight of them, and so the possibility cannot be ruled out that misclassification may have occurred in some cases. Although the described association of immune response with a status of latency is highly likely, the numbers of individuals enrolled in the present proof-of-principle study are limited. This indicates the need to confirm the data in larger independent studies. Moreover, the groups enrolled were not fully matched for region of origin. In order to evaluate whether or not the results could have been influenced by differences in the ethnic distribution of the various groups, the analysis was repeated, including only those who originated from Western European countries, which represented the largest population group. In this group, the same associations 
observed in the overall population were found (data not shown). This observation suggests that the present results are most probably not biased by ethnicity or country of origin. These results were obtained by evaluating the response to Rv2628 at a single time-point; however, an evaluation of those with active TB and recent infection over time is crucial in order to understand the meaning of the present study in a clinical context, and ongoing studies are in progress. Finally, several comparisons were performed in order to assess the possible association of each antigen with stage of TB, and this may have increased the chance of false-positive associations. However, the differences in response to Rv2628 between those able to control $M$. tuberculosis replication, either naturally (remote LTBI) or following chemotherapy (cured TB), and those with active $M$. tuberculosis replication, either controlled, but acute (recent infection), or uncontrolled (active disease), were still highly significant after applying the correction for multiple comparisons.

In conclusion, a whole-blood short-term IFN- $\gamma$ response to Rv2628 is frequently detected in remote LTBI, but is rarely found in recently acquired infection. These results may have an important practical clinical implication for risk stratification when deciding to initiate preventive therapy for LTBI.

\section{SUPPORT STATEMENT}

This research was supported by grants from Funds for Current Intramural Research and Research Program Project Grant 06.76.1 and 07.103 (Italian Ministry of Health, Rome, Italy), the European Commission (Brussels, Belgium) and the Bill and Melinda Gates Foundation Grand Challenges in Global Health program (Seattle, WA, USA).

\section{STATEMENT OF INTEREST}

Statements of interest for E. Girardi, D. Goletti and T.H.M. Ottenhoff and the study itself can be found at www.eri.ersjournals.com/misc/ statements.dtl

\section{ACKNOWLEDGEMENTS}

The authors are grateful to all of the patients and nurses (in particular M. Vecchi and C. Copertino (both Pneumology Division, National Institute for Infectious Diseases L. Spallanzani (INMI), Rome, Italy) and G. Cuzzi (Epidemiology Dept, INMI)) who took part in the study. We are grateful to A. Baker (Scientific Direction, INMI) for the editing.

\section{REFERENCES}

1 World Health Organization, Global Tuberculosis Control 2008 Surveillance, Planning, Financing. Geneva, World Health Organization, 2008.

2 Pai M, Zwerling A, Menzies D. Systematic review: T-cell-based assays for the diagnosis of latent tuberculosis infection: an update. Ann Intern Med 2008; 149: 177-184.

3 Lalvani A. Diagnosing tuberculosis infection in the 21st century: new tools to tackle an old enemy. Chest 2007; 131: 1898-1906.

4 Ferrara G, Losi M, Meacci M, et al. Routine hospital use of a new commercial whole blood interferon- $\gamma$ assay for the diagnosis of tuberculosis infection. Am J Respir Crit Care Med 2005; 172: 631-635.

5 Goletti D, Stefania C, Butera O, et al. Accuracy of immunodiagnostic tests for active tuberculosis using single and combined results: a multicenter TBNET-Study. PLoS One 2008; 3: e3417.

6 Goletti D, Carrara S, Vincenti D, et al. Accuracy of an immune diagnostic assay based on RD1 selected epitopes for active tuberculosis in a clinical setting: a pilot study. Clin Microbiol Infect 2006; 12: 544-550.

7 Goletti D, Parracino MP, Butera O, et al. Isoniazid prophylaxis differently modulates T-cell responses to RD1-epitopes in contacts recently exposed to Mycobacterium tuberculosis: a pilot study. Respir Res 2007; 8: 5.

8 Marais BJ, Gie RP, Schaaf HS, et al. Childhood pulmonary tuberculosis: old wisdom and new challenges. Am J Respir Crit Care Med 2006; 173: 1078-1090

9 Ulrichs T, Kosmiadi GA, Trusov V, et al. Human tuberculous granulomas induce peripheral lymphoid follicle-like structures to orchestrate local host defence in the lung. J Pathol 2004; 204 217-228.

10 Wayne LG, Hayes LG. Nitrate reduction as a marker for hypoxic shiftdown of Mycobacterium tuberculosis. Tuber Lung Dis 1998; 79: 127-132.

11 Tufariello JM, Chan J, Flynn JL. Latent tuberculosis: mechanisms of host and bacillus that contribute to persistent infection. Lancet Infect Dis 2003; 3: 578-590.

12 Yuan Y, Crane DD, Barry CE III. Stationary phase-associated protein expression in Mycobacterium tuberculosis: function of the mycobacterial $\alpha$-crystallin homolog. J Bacteriol 1996; 178: 4484-4492.

13 Park HD, Guinn KM, Harrell MI, et al. Rv3133c/dosR is a transcription factor that mediates the hypoxic response of Mycobacterium tuberculosis. Mol Microbiol 2003; 48: 833-843.

14 Leyten EM, Lin MY, Franken KL, et al. Human T-cell responses to 25 novel antigens encoded by genes of the dormancy regulon of Mycobacterium tuberculosis. Microbes Infect 2006; 8: 2052-2060.

15 Schuck SD, Mueller H, Kunitz F, et al. Identification of T-cell antigens specific for latent Mycobacterium tuberculosis infection PLoS One 2009; 4: e5590.

16 Black GF, Thiel BA, Ota M, et al. Immunogenicity of novel DosR regulon-encoded candidate antigens of Mycobacterium tuberculosis in three high-burden populations in Africa. Clin Vaccine Immunol 2009; 16: 1203-1212.

17 Weir RE, Gorak-Stolinska P, Floyd S, et al. Persistence of the immune response induced by BCG vaccination. BMC Infect Dis 2008; 8: 9 .

18 Lawn SD, Bangani N, Vogt $\mathrm{M}$, et al. Utility of interferon- $\gamma$ ELISPOT assay responses in highly tuberculosis-exposed patients with advanced HIV infection in South Africa. BMC Infect Dis 2007; 7: 99.

19 Geluk A, Lin MY, van Meijgaarden KE, et al. T-cell recognition of the HspX protein of Mycobacterium tuberculosis correlates with latent $M$. tuberculosis infection but not with $M$. bovis BCG vaccination. Infect Immun 2007; 75: 2914-2921.

20 National Collaborating Centre for Chronic Conditions. Tuberculosis Clinical Diagnosis and Management of Tuberculosis, and Measures for its Prevention and Control. London, Royal College of Physicians, 2006.

21 Franken KL, Hiemstra HS, van Meijgaarden KE, et al. Purification of His-tagged proteins by immobilized chelate affinity chromatography: the benefits from the use of organic solvent. Protein Expr Purif 2000; 18: 95-99.

22 Diagnostic standards and classification of tuberculosis in adults and children. Am J Respir Crit Care Med 2000; 161: 1376-1395.

23 Voskuil MI, Schnappinger D, Visconti KC, et al. Inhibition of respiration by nitric oxide induces a Mycobacterium tuberculosis dormancy program. J Exp Med 2003; 198: 705-713.

24 Schnappinger D, Ehrt S, Voskuil MI, et al. Transcriptional adaptation of Mycobacterium tuberculosis within macrophages: insights into the phagosomal environment. J Exp Med 2003; 198: 693-704.

25 Demissie A, Leyten EM, Abebe M, et al. Recognition of stagespecific mycobacterial antigens differentiates between acute and 
latent infections with Mycobacterium tuberculosis. Clin Vaccine Immunol 2006; 13: 179-186.

26 Roupie V, Romano M, Zhang L, et al. Immunogenicity of eight dormancy regulon-encoded proteins of Mycobacterium tuberculosis in DNA-vaccinated and tuberculosis-infected mice. Infect Immun 2007; 75: 941-949.

27 Shi L, Jung YJ, Tyagi S, et al. Expression of Th1-mediated immunity in mouse lungs induces a Mycobacterium tuberculosis transcription pattern characteristic of nonreplicating persistence. Proc Natl Acad Sci USA 2003; 100: 241-246.

28 Boon C, Li R, Qi R, et al. Proteins of Mycobacterium bovis BCG induced in the Wayne dormancy model. J Bacteriol 2001; 183: 2672-2676.
29 Mayuri, Bagchi G, Das TK, et al. Molecular analysis of the dormancy response in Mycobacterium smegmatis: expression analysis of genes encoding the DevR-DevS two-component system, Rv3134c and chaperone alpha-crystallin homologues. FEMS Microbiol Lett 2002; 211: 231-237.

30 Lin MY, Reddy TK, Arend SM, et al. Cross-reactive immunity to Mycobacterium tuberculosis DosR regulon-encoded antigens in individuals infected with environmental, nontuberculous mycobacteria. Infect Immun 2009; 77: 5071-5079.

31 Lin MY, Ottenhoff TH. Host-pathogen interactions in latent Mycobacterium tuberculosis infection: identification of new targets for tuberculosis intervention. Endocr Metab Immune Disord Drug Targets 2008; 8: 15-29. 